\title{
NOTE ON THE BOREL METHOD \\ OF MEASURE EXTENSION
}

\author{
G. Fox
}

(received May 22, 1962)

Introduction. This note concerns a countably additive measure on a Boolean ring of subsets of an abstract set, this measure being real-valued, admitting $\infty$ as a possible value. We a re interested only in unique extensions, so we suppose the measure to be $\sigma$-finite. The following well known result will be referred to as the "extension theorem": "Every $\sigma$-finite measure on a ring extends uniquely to a $\sigma$-finite measure on the generated $\sigma$-ring." Besides the familiar proof using outer measure, the re is a Borel-type proof using transfinite induction [4]. We attempt here to reduce the Borel-type proof to its ultimate simplicity, reducing the problem to the bounded case.

Proof for a bounded measure. In this section $\mu$ is a measure (1) on a ring $R$ (of subsets of a fixed abstract set $X$ ) which is bounded above by a fixed real constant $M$ : $0 \leq \mu(\mathrm{R}) \leq \mathrm{M}<\infty$, all $\mathrm{R} \in \mathcal{R}$. The boundedness of $\mu$ will be applied in the transfinite induction.

If a sequence $R_{n}, n=1,2, \ldots\left(R_{n} \in R\right)$ converges settheoretically to $R \in R$, then $\mu\left(R_{n}\right), n=1,2, \ldots$ converges to $\mu(R)$. This is expressed symbolically:

(1)

$$
\mathrm{R}_{\mathrm{n}} \rightarrow \mathrm{R} \Rightarrow \mu\left(\mathrm{R}_{\mathrm{n}}\right) \rightarrow \mu(\mathrm{R}) \quad\left(\mathrm{R}, \mathrm{R}_{\mathrm{n}} \in R\right)
$$

(1) The term "measure" will imply countable additivity.

Canad. Math. Bull. vol. 5, no. 3, September 1962. 
Assuming the extension theorem, (1) is a consequence of the countable additivity; but we will prove it independently, then use it as basis for proof of the extension theorem. It will suffice to prove the particular case:

$$
R_{n} \rightarrow 0 \Rightarrow \mu\left(R_{n}\right) \rightarrow 0 \quad\left(R_{n} \in K^{\prime}\right) .
$$

In fact, the hypothesis of (1) asserts ${ }^{(2)}$ that $R_{n} \Delta R \rightarrow 0$, and applying (2),

$$
\mu\left(R_{n}\right)-\mu(R) \leq \mu\left(R_{n}-R\right) \leq \mu\left(R_{n} \Delta R\right) \rightarrow 0
$$

But this implies $\operatorname{Iim} \sup \mu\left(R_{n}\right) \leq \mu(R)$, and we show similarly that $\lim \inf \mu\left(R_{n}\right) \geq \mu(R)$.

To prove (2), assume its hypothesis, and set $S_{n}=\underset{i=n}{\cup} R_{i}$, so that (3) $R_{n} \subseteq S_{n} \downarrow 0$. The $S_{n}$ need not belong to $F_{i}$, but it will be shown in the next paragraph that they may be approximated by members of $i$ as follows: To a rbitrary $\epsilon>0$ there corresponds a sequence $T_{n}, n=1,2, \ldots$ such that

$$
\begin{aligned}
& \text { (i) } T_{n} \in R, S_{n} \supseteq T_{n} \downarrow 0 \\
& \text { (ii) } S_{n} \supseteq R \in R \Rightarrow \mu(R)-\mu\left(T_{n}\right) \leq \in \sum_{i=1}^{n} \frac{1}{2^{i}} .
\end{aligned}
$$

Assuming this, $\mu\left(T_{n}\right) \downarrow 0$ by (i), then $\operatorname{Iim} \sup \mu\left(R_{n}\right) \leq \epsilon$ by (ii), and since $\epsilon$ is a rbitrary (2) will be proved, hence also (1).

(2) The symmetric difference $E \Delta F=(E-F) \cup(F-E)$ is the ring addition, intersection being the ring multiplication.

(3) The symbols $\uparrow, \downarrow$ indicate monotone increasing, decreasing convergence. 
To establish the sequence $T_{n}$, set $E_{n}=\underset{i=n}{\bigcup} R_{i}$ where $n^{\prime}$ is a fixed index $\geq n$ of sufficiently high rank that

$$
\mu\left(\underset{i=n}{\cup} R_{i}\right)-\mu\left({\underset{i=n}{n^{\prime}}}_{i}^{\cup} R_{i}\right)<\frac{\epsilon}{2^{n}} \text { for all } m \geq n^{\prime} .
$$

The $T_{n}$ are defined inductively:

$$
T_{1}=E_{1}, \quad T_{n}=E_{n} \cap T_{n-1} \text { for } n>1
$$

Since $S_{n} \supseteq E_{n} \supseteq T_{n}$ (i) is satisfied, and (ii) is satisfied for $\mathrm{n}=1$. In fact, if $\mathrm{S}_{1} \supseteq \mathrm{R} \in \mathcal{R}$ then $\mathrm{R} \cap \underset{\mathrm{i}=1}{\mathrm{U}} \mathrm{R}_{\mathrm{i}}, \mathrm{m}=1,2, \ldots$ increases monotonely to $R$, so that

$\mu(R)-\mu\left(T_{1}\right)=\lim _{m \rightarrow \infty} \mu\left(R \cap V_{i=1} R_{i}\right)-\mu\left(E_{1}\right) \leq \lim _{m \rightarrow \infty} \mu\left(\cup_{i=1}^{\cup} R_{i}\right)-\mu\left(E_{1}\right) \leq \frac{\epsilon}{2}$.

It remains to show that (ii) is satisfied for $n$, assuming it for n-1. Assuming the hypothesis of (ii),

$\mu(R)-\mu\left(E_{n}\right)=\lim _{m \rightarrow \infty} \mu\left(R_{i=n} \cup R_{i}\right)-\mu\left(E_{n}\right) \leq \lim _{m \rightarrow \infty} \mu\left(\cup_{i=n}^{m} R_{i}\right)-\mu\left(E_{n}\right) \leq \frac{\epsilon}{2^{n}}$

$\mu\left(E_{n}\right)-\mu\left(T_{n}\right)=\mu\left(E_{n}-T_{n}\right)=\mu\left(E_{n}-T_{n-1}\right)=\mu\left(E_{n} \cup T_{n-1}\right)-\mu\left(T_{n-1}\right)$.

Since $E_{n} \subseteq S_{n} \subseteq S_{n-1}$ and the refore $S_{n-1} \supseteq E_{n} \cup T_{n-1} \in$ 不, by the induction hypothesis,

$$
\mu\left(E_{n} \cup T_{n-1}\right)-\mu\left(T_{n-1}\right) \leq \in \sum_{i=1}^{n-1} \frac{1}{2^{i}}
$$

The combined inequalities give the right member of (ii), thus completing the induction and so establishing (1). 
The definition of set-theoretical convergence carries over, without essential modification, to Moore-Smith sequences of sets. For double-index sequences we have the following analogue of (2):

$$
\mathrm{R}_{\mathrm{mn}} \rightarrow 0 \Longrightarrow \mu\left(\mathrm{R}_{\mathrm{mn}}\right) \rightarrow 0 \quad\left(\mathrm{R}_{\mathrm{mn}} \in R\right)
$$

In fact, if the right member of (3) were false there would exist $\epsilon>0$ and two subsequences of the positive integers:

$\mathrm{m}_{1}<\mathrm{m}_{2}<\ldots, \mathrm{n}_{1}<\mathrm{n}_{2}<\ldots$ such that $\mu\left(\mathrm{R}_{\mathrm{m}_{\mathrm{k}} \mathrm{n}_{\mathrm{k}}}\right) \geq \epsilon$ for

$k=1,2, \ldots$, But because of (2) this would contradict the convergence $\lim R, \quad=0$ implied by the left member of (3). ${ }_{k \rightarrow \infty} m_{k} n_{k}$

We now show that if $R_{n} \rightarrow E\left(R_{n} \in \mathcal{K}\right)$, then $\mu\left(R_{n}\right)$, $\mathrm{n}=1,2, \ldots$ converges to a limit which depends only on the limit set $E$. Applying (3), we establish $\mu\left(R_{n}\right), n=1,2, \ldots$ as a Cauchy sequence:

$$
\begin{aligned}
\left|\mu\left(R_{m}\right)-\mu\left(R_{n}\right)\right| & =\max \left\{\mu\left(R_{m}\right)-\mu\left(R_{n}\right), \mu\left(R_{n}\right)-\mu\left(R_{m}\right)\right\} \\
& \leq \max \left\{\mu\left(R_{m}-R_{n}\right), \mu\left(R_{n}-R_{m}\right)\right\} \\
& \leq \mu\left(R_{m}-R_{n}\right)+\mu\left(R_{n}-R_{m}\right)=\mu\left(R_{m} \Delta R_{n}\right) \rightarrow 0 .
\end{aligned}
$$

Given a second sequence converging to the same limit: $R$ $R_{n}^{\prime} \rightarrow E\left(R_{n}^{\prime} \in \wedge^{\prime}\right)$, we have $R_{n}^{\prime} \Delta R_{n} \rightarrow 0$, so that

$$
\mu\left(R_{n}^{\prime}\right)-\mu\left(R_{n}\right) \leq \mu\left(R_{n}^{\prime}-R_{n}\right) \leq \mu\left(R_{n}^{\prime} \Delta R_{n}\right) \rightarrow 0
$$

Therefore $\operatorname{Iim} \mu\left(R_{n}^{\prime}\right) \leq \operatorname{Iim} \mu\left(R_{n}\right)$ and, by symmetry, we have the inverse inequality, completing the proof of the assertion.

Let $R *$ be the class of limits of convergent sequences $\mathrm{R}_{\mathrm{n}}, \mathrm{n}=1,2, \ldots\left(\mathrm{R}_{\mathrm{n}} \in R\right)$; this is a ring extension of $R$, which we refer to as the "Iimit ring" of $R$. The assertion of the preceding paragraph implies that a function $\mu *$ is well defined on $K^{\prime} *$ by the formula: 


$$
\mu *(\mathrm{R} *)=\lim \mu\left(\mathrm{R}_{\mathrm{n}}\right) \text {, whe re } \mathrm{R}_{\mathrm{n}} \rightarrow \mathrm{R} *\left(\mathrm{R}_{\mathrm{n}} \in R, \mathrm{R} * \in \Lambda^{*}\right)
$$

It follows from (1) that $\mu$ extends $\mu$, and that any measure extension of $\mu$ to $R_{*}^{*}$ must coincide with $\mu *$. To prove the finite additivity of $\mu^{*}$, consider a disjoint union(4) of two members of $R *: R *+S *$. We have

$$
R_{n} \rightarrow R^{*}, \quad S_{n} \rightarrow S^{*}, \quad R_{n} \cap S_{n} \rightarrow 0 \quad\left(R_{n}, S_{n} \in R_{1}\right)
$$

Applying (2),

$\mu *(R *+S *)=\lim \mu\left(R_{n} \cup S_{n}\right)=\operatorname{Iim}\left(\mu\left(R_{n}\right)+\mu\left(S_{n}\right)+\mu\left(R_{n} \cap S_{n}\right)\right)=\mu *(R *)+\mu *(S *)$.

So $\mu *$ will be a measure if we show, further, that

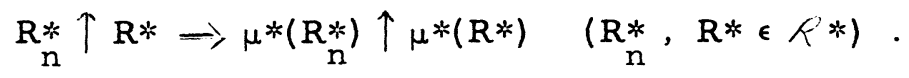

In order to prove (4) we introduce the class $U /$ consisting of all finite or countable unions of members of $K$ :

$K \subseteq U \subseteq K^{*}$. We begin by proving that

$$
U_{n} \uparrow U \Rightarrow \mu *\left(U_{n}\right) \uparrow \mu *(U) \quad\left(U_{n}, U \in U\right)
$$

We have: $U_{\mathrm{n}}=\bigcup_{\mathrm{m}} \mathrm{R}_{\mathrm{nm}}\left(\mathrm{R}_{\mathrm{nm}} \in R\right), \quad \mathrm{U}=\bigcup_{\mathrm{n}} \underset{\mathrm{m}}{\cup} \mathrm{R}_{\mathrm{nm}}$. We may re-order the $R_{m n}$ as terms of a simple sequence (with indices $1,2, \ldots$ ) then take the partial (finite) unions as the terms $R_{n}$ of a sequence increasing to $U$ :

$$
\mathrm{R}_{\mathrm{n}} \uparrow U\left(\mathrm{R}_{\mathrm{n}} \in R\right), \quad \mu\left(\mathrm{R}_{\mathrm{n}}\right) \uparrow \mu *(U)
$$

Then (5) follows from the fact that each $R_{n}$ is contained in some $U_{m}$. Now assume the hypothesis of (4), and let $\epsilon>0$ be arbitrary. Considering $\underset{n}{R *}$ as the superior limit of a sequence converging to $\underset{n}{*}$ whose terms belong to $R$, we see

$\overline{(4) \text { We substitute }+ \text { or } \Sigma}$ for $\cup$ to indicate a disjoint union. 
that there exist sets $U_{n} \in U$ such that

$$
U_{n} \supseteq \underset{n}{R *} \text { and } \mu *\left(U_{n}\right)-\mu *\left(R_{n}^{*}\right)<\frac{\epsilon}{2^{n}}
$$

Then $U_{n}^{\prime}=\bigcup_{i=1}^{n} U_{i}, n=1,2, \ldots$ is an increasing sequence of sets of $U$ having a Iimit $U \in U$, so by (5),

$$
\mu^{* *}\left(U_{n}^{\prime}\right) \uparrow \mu^{* *}(U) \geq \mu^{* *}(R *)
$$

But $\left.\mu *\left(U_{n}^{\prime}\right)-\mu * \underset{n}{R *}\right)=\mu *\left(\bigcup_{i=1}^{n} U_{i}-R_{n}^{*}\right) \leq \sum_{i=1}^{*} \mu^{*}\left(U_{i}-R_{i}^{*}\right)<\epsilon$.

Then, since $\epsilon$ is arbitrary, $\lim \mu *\left(R_{n}^{*}\right) \geq \mu *(R *)$, and the inverse inequality is obvious, proving (4). This establishes $\mu$ * as the unique measure extension to $R$ * of the original measure $\mu$ on $R$.

We define inductively the transfinite sequence of rings:

$$
N^{\prime}=R_{0} \subset R_{1} \subset K_{2} \subset \ldots \subset R_{\alpha} \subset \ldots \subset R_{\sigma}
$$

For $\alpha>0$, an ordinal of the first kind, $R_{\alpha}=R_{\alpha-1}^{*}$, the limit ring of $K_{\alpha-1}$. For an ordinal $\alpha$ of the second kind, $\hat{R}_{\alpha}=\bigcup_{\beta<\alpha} R_{\beta}$. The ordinal $\sigma$ is the smallest such that $R_{\sigma}^{*}=\sigma_{\sigma}$, so $R_{\sigma}$ is the $\sigma$-ring generated by $R$. (The symbol $\sigma$ denotes this ordinal and also serves as the sign of the generated $\sigma$-ring.) In the case $\sigma=0, K^{\prime}$ is already an $\sigma$-ring and there is no measure extension problem. An ordinal $\alpha(0 \leq \alpha \leq \sigma)$ will be called "accessible" if

(i) The original measure $\mu$ on $P$ extends uniquely to a measure $\mu_{\alpha}$ on $R_{\alpha}, \mu_{\alpha}$ being bounded by $M$, the bound of $\mu$.

(ii) For arbitrary $\epsilon>0$ and $S \in \mathcal{R}_{\alpha}$, there exists $U \in \mathcal{U}$ (class of finite or countable unions of members of $R$ ) such that 


$$
U \supseteq S \text { and } \mu_{\alpha}(U)-\mu_{\alpha}(S)<\epsilon \text {. }
$$

To complete the proof of the extension theorem for the bounded case, it will suffice to prove inductively that every $\alpha$ $(0 \leq \alpha \leq \sigma)$ is accessible. Since 0 is accessible, it suffices to prove $\alpha>0$ accessible, assuming every $\beta<\alpha$ accessible.

First, let $\alpha$ be of the first kind so that $\alpha-1$ is accessible. As has been shown, $\mu_{\alpha-1}$ extends uniquely to a measure $\mu_{\alpha}$ on $\ell_{\alpha-1}^{*}=K_{\alpha}$ which is obviously bounded by $M$. But $\mu_{\alpha}$, the unique measure extension of $\mu_{\alpha-1}$, itself the unique measure extension of $\mu$ to $\hat{h}_{\alpha-1}$, is the unique measure extension of $\mu$ to $R_{\alpha}$, so (i) is satisfied for $\alpha$. The class $T_{\alpha-1}$ of finite or countable unions of members of $\kappa_{\alpha-1}$, is related to $P_{\alpha}$ as is $Z_{1}$ to $K_{1}$. So, for arbitrary

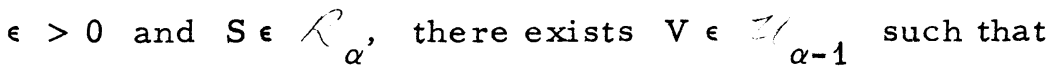

$$
\mathrm{V} \supseteq \mathrm{S} \text { and } \mu_{\alpha}(\mathrm{V})-\mu_{\alpha}(\mathrm{S})<\frac{\epsilon}{2}
$$

By the definition of $Z_{\alpha-1}$ and the fact that $P_{\alpha-1}$ is a ring, we may express $V$ as a disjoint finite or countable union of members of $R_{\alpha-1}$ :

$$
V=\Sigma T_{n}\left(T_{n} \in F_{\alpha-1}\right)
$$

Since $\alpha-1$ is accessible and $\mu_{\alpha}$ extends $\mu_{\alpha-1}$, the re exists $\mathrm{U}_{\mathrm{n}} \in Z_{\text {I such that }}$

$$
U_{n} \supseteq T_{n} \text { and } \mu_{\alpha}\left(U_{n}\right)-\mu_{\alpha}\left(T_{n}\right)<\frac{\epsilon}{2^{n+1}} \text {. }
$$

$$
\begin{aligned}
& \text { Setting } U=\bigcup_{n} U_{n} \text {, we have } S \subseteq V \subseteq U \in \mathbb{Z} \\
& \mu_{\alpha}(U)-\mu_{\alpha}(S)=\mu_{\alpha}(U)-\mu_{\alpha}(V)+\mu_{\alpha}(V)-\mu_{\alpha}(S)<\mu_{\alpha}(U)-\mu_{\alpha}(V)+\frac{\epsilon}{2} .
\end{aligned}
$$


But since $\mu_{\alpha}$ is a finite measure on $\mathcal{K}_{\alpha}$,

$\left.\mu_{\alpha}(U)-\mu_{\alpha}(V)=\mu_{\alpha}(U-V) \leq \mu_{\alpha}\left\{\bigcup_{n} U_{n}-T_{n}\right)\right\} \leq \Sigma \mu_{\alpha}\left(U_{n}-T_{n}\right)<\frac{\epsilon}{2}$.

The combined inequalities establish (ii) for $\alpha$.

Now let $\alpha$ be of the second kind. Because of the induction hypothesis, a function $\mu_{\alpha}$ is well defined on $\wedge_{\alpha}$ by the formula $\mu_{\alpha}(S)=\mu_{\beta}(S)$, where $\beta<\alpha$ is such that $S \in K_{\beta}$ $\left(S \in A_{\alpha}\right)$. It must be shown that, in virtue of this definition, $\alpha$ is accessible. Already, by the induction hypothesis, (ii) is satisfied, $\mu_{\alpha}$ is finitely additive and bounded by $M$. Since any measure extension of $\mu$ to $\gamma_{\alpha}$ would satisfy the defining formula for $\mu_{\alpha}, \mu_{\alpha}$ is the only possible such extension. It remains only to prove the countable additivity of $\mu_{\alpha}$. Suppose that $S=\sum_{1}^{\infty} S_{n}\left(S, S_{n} \in \swarrow_{\alpha}\right)$. Because of the finite additivity, $\mu_{\alpha}(S) \geq \sum_{1}^{\infty} \mu_{\alpha}\left(S_{n}\right)$

Applying (ii) for $\alpha$, for given $\epsilon>0$ there exist sets $U_{n} \epsilon$ such that

$$
U_{n} \supseteq S_{n} \quad \text { and } \quad \mu_{\alpha}\left(U_{n}\right)-\mu_{\alpha}\left(S_{n}\right)<\frac{\epsilon}{2^{n}} .
$$

Because $\mu_{\alpha}$, restricted to $\Re_{1}$, is the measure $\mu_{1}$,

$$
\mu_{\alpha}(S) \leq \mu_{\alpha}\left(\begin{array}{l}
\infty \\
\cup \\
1
\end{array}\right) \leq \sum_{1}^{\infty} \mu_{\alpha}\left(U_{n}\right)<\sum_{1}^{\infty} \mu_{\alpha}\left(S_{n}\right)+\epsilon .
$$

Since $\epsilon$ is a rbitrary the combined inequalities give

$$
\mu_{\alpha}(S)=\sum_{1}^{\infty} \mu_{\alpha}\left(S_{n}\right) \text {. }
$$

This completes the induction, and therefore the proof of the extension theorem for the bounded case. 
Proof for a $\sigma$-finite measure. Henceforth $\mu$ is a $\sigma$-finite measure on the ring $R$. Applying the extension theorem for the bounded case, we show in this section that the $\sigma$-finite measure $\mu$ extends uniquely to a $\sigma$-finite measure on the generated $\sigma$-ring $K_{\sigma}$. Let $\zeta_{0}$ be the subring of $K^{\prime}$ consisting of the sets of $K^{\prime}$ of finite measure. Because $\mu$ is $\sigma$-finite the $\sigma$-ring generated by $\zeta_{0}$ is $\zeta^{\prime} \sigma$ Therefore to extend $\mu$ uniquely to a measure on $A_{\sigma}$ it will suffice to do the same for its restriction to $Y_{0}$. So we assume henceforth, without loss of generality, that the original measure $\mu$ on $K$ is finite.

Between $\Lambda^{\prime}$ and $\uparrow_{\sigma}$ we interpolate the ring $/$ ' consisting of all members of $\lambda_{\sigma}$ contained in some member

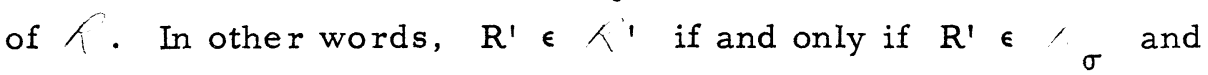
$R^{\prime} \subseteq R$ for some $R \in /$ (depending on $R^{\prime}$ ). Let $\bigwedge_{1}$ be a given ring (of subsets of $X$ ) and let $E$ be a given set (subset of $\mathrm{X}$ ). The "trace" of $K_{1}$ on $\mathrm{E}$, denoted $\overbrace{1} \cap \mathrm{E}$, is the ring of sets of the form $R_{1} \cap \mathrm{E}$, where $R_{1} \in / /_{1}$. Since a member of $T_{\sigma}$ is contained in $R \in /$ if and only if it belongs to the trace $Y_{\sigma} \cap \mathrm{R}$, we have

$$
K^{\prime}=\bigcup_{\mathrm{R} \in R}\left(K_{\sigma} \cap \mathrm{R}\right), \mathrm{R} \subseteq \mathbb{R}^{\prime} \subseteq K_{\sigma}^{\prime}
$$

Our procedure will be to first extend $\mu$ uniquely to a finite measure $\mu^{\prime}$ on $\kappa^{\prime}$, then to extend $\mu^{\prime}$ uniquely to a $\sigma$-finite measure on $K_{\sigma}$.

First, we extend $\mu$ uniquely to a finite measure $\mu^{\prime}$ on $R^{\prime}$. For arbitrary $R \in K$, the restriction of $\mu$ to $\cap \cap R$ is bounded, and the $\sigma$-ring generated by $R \cap R$ is (5)

$$
(R \cap \mathrm{R})_{\sigma}=R_{\sigma} \cap \mathrm{R} .
$$

(5) The left member is merely the notation for the generated $\sigma$-ring. Its identity with the right member is proved as theorem E, sec. 5 [3]. 
The extension theorem for the bounded case asserts that this restriction extends uniquely to a finite measure $\mu_{\mathrm{R}}$ on $K_{\sigma} \frown R$. We will combine the measures $\mu_{R}, R \in K$ to form a single measure $\mu^{\prime}$ on $K^{\prime} '$. We observe that if $R_{1} \subseteq R_{2}\left(R_{1}, R_{2} \in \curlywedge^{\prime}\right)$ then $\mu_{R_{1}}$ and the restriction of $\mu_{R_{2}}$ to ${ }_{\sigma} \cap R_{1}$ must coincide on their common domain, since they are both measure extensions of the restriction of $\mu$ to A' $\frown R_{1}$ (uniqueness of measure extension in the bounded case). Hence, for any two members $R_{1}, R_{2}$ of $N^{\prime}, \mu_{R_{1}}, \mu_{R_{2}}$ coincide on ${ }_{\sigma} \cap\left(R_{1} \frown R_{2}\right)$. Therefore a function $\mu^{\prime}$ is weil defined on $A$ ' by the formula $\mu^{\prime}\left(R^{\prime}\right)=\mu_{R}\left(R^{\prime}\right)$, where $R$ is such that $R^{\prime} \cong R \in R\left(R^{\prime} \in R^{\prime}\right)$.

It is already obvious that $\mu^{\prime}$ is a finite measure on $R^{\prime}$ which extends every $\mu_{R}$, and so also extends $\mu$. But any measure on $\lambda^{\prime}$ ' which extends $\mu$ must also extend every $\mu_{R}\left(R \in h^{\prime}\right)$ (uniqueness of measure extension in the bounded case), so $\mu^{\prime}$ is the only possible measure extension of $\mu$ to $A^{\prime}$ '.

We now extend $\mu^{\prime}$ uniquely to a $\sigma$-finite measure on ${ }^{\prime} \sigma$. Every member of $1_{\sigma}$ may be expressed as a disjoint, finite or countable, union of members of 1 . Consider two such representation $s$ of the same set $E \in K_{\sigma}^{\prime}$ :

$$
E=\Sigma S_{n}=\Sigma T_{m} \quad\left(S_{n}, T_{m} \in R^{\prime}\right)
$$

Since $\mu^{\prime}$ is a measure on $\kappa^{\prime}$,

$$
\underset{\mathrm{n}}{\sum_{\mu}}\left(\mathrm{S}_{\mathrm{n}}\right)=\sum_{\mathrm{n}} \mu^{\prime}\left(\mathrm{S}_{\mathrm{n}} \frown \underset{\mathrm{m}}{\sum_{\mathrm{m}}} \mathrm{T}_{\mathrm{n}}\right)=\underset{\mathrm{m}}{\sum_{\mathrm{m}}} \mu^{\prime}\left(\mathrm{S}_{\mathrm{n}} \frown \mathrm{T}_{\mathrm{m}}\right)=\underset{\mathrm{m}}{\sum} \mu^{\prime}\left(\mathrm{T}_{\mathrm{m}}\right) .
$$

Therefore a function $\mu_{\sigma}$ is well defined on $K_{\sigma}^{\prime}$ by the formula $\mu_{\sigma}(E)=\Sigma \mu^{\prime}\left(S_{n}\right)$, where $E=\Sigma S_{n}\left(S_{n} \in K^{\prime}\right)$ is any representation 
The function $\mu_{\sigma}$ extends $\mu^{\prime}$, it is finitely additive and, if it is shown to be a measure, it will be $\sigma$-finite. But any measure on $R_{\sigma}$ extending $\mu^{\prime}$ must satisfy the defining equation for $\mu_{\sigma}$, so $\mu_{\sigma}$ is the only possible such extension. It remains only to prove the countable additivity fof $\mu_{\sigma}$. Suppose that

$$
E=\sum_{1}^{\infty} E_{n} \quad\left(E_{n} \in R_{\sigma}\right)
$$

Each term of the union has a representation of the form ${ }^{(6)}$

$$
E_{n}=\sum_{m=1}^{\infty} s_{n m} \quad\left(s_{n m} \in R^{\prime}\right)
$$

In the special case where $E \in R^{\prime}$ we have

$\mu_{\sigma}(E)=\mu^{\prime}(E)=\mu^{\prime}\left(\sum_{n=1}^{\infty} \sum_{m=1}^{\infty} S_{n m}\right)=\sum_{n=1}^{\infty} \sum_{m=1}^{\infty} \mu^{\prime}\left(S_{n m}\right)=\sum_{n=1}^{\infty} \mu_{\sigma}\left(E_{n}\right)$.

In the general case we may express $E: E=\sum_{1}^{\infty} S_{n}\left(S_{n} \in R^{\prime}\right)$.

We apply the special case, taking account of the definition $\mu_{\sigma}$, and noting that $\mathrm{S}_{\mathrm{n}} \cap \mathrm{E}_{\mathrm{m}} \in R^{\prime}$ :

$$
\begin{aligned}
\mu_{\sigma}(E) & =\sum_{n=1}^{\infty} \mu^{\prime}\left(S_{n}\right)=\sum_{n=1}^{\infty} \mu^{\prime}\left(S_{n} \cap \sum_{m=1}^{\infty} E_{m}\right)=\sum_{n=1}^{\infty} \sum_{m=1}^{\infty} \mu^{\prime}\left(S_{n} \cap E_{m}\right) \\
& =\sum_{m=1}^{\infty} \sum_{n=1}^{\infty} \mu^{\prime}\left(S_{n} \cap E_{m}\right)=\sum_{m=1}^{\infty} \mu_{\sigma}\left(E_{m}\right) .
\end{aligned}
$$

This establishes the countable additivity of $\mu_{\sigma}$, and so completes our proof of the extension theorem.

(6) By adjoining, if necessary, an infinity of terms equal to the empty set, we may suppose all representations to be countably infinite. 


\section{REFERENCES}

1. J. Albuque rque, Ensembles de Borel, Portugaha Math. (1944).

2. E. Borel, Leçons sur la théorie des fonctions. Collection de monographies sur la théorie des fonctions publiées sous la direction de Emile Borel (4e éd Paris, Gauthier-Villars 19!

3. P. R. Halmos, Measure Theory (New York, 1950).

4. L. LeBlanc et G.E. Fox, On the Extension of Measure by the Method of Borel. Can. Jour. of Math. vol. 8 , pp. 516-523, 1956.

5. N. Lusin, Leçons sur les ensembles analytiques et leurs applications. Collection de monographies sur la théorie des fonctions publiées sous la direction de Emile Borel (Paris, 1930).

6. R. Neves, Sôbre a construçae algebrica da teoria ge ral da Melida. Instituto Para a alta cultura, Gendro de estudas matematicas, Publ.no.13, 22 pp. (1945).

Université de Montréal 